\title{
Cirugía oral en pacientes bajo terapia anticoagulante: modelo experimental para la evaluación de la eficacia de la cola de fibrina
}

\author{
Taormina G*, Papagna R*, Carini F*, Denotti G**, Calzavara D***
}

\section{RESUMEN}

Introducción: En la práctica de cirugía oral en los pacientes bajo terapia anti coagulante, el empleo de un sistema eficaz de acción hemostática es de extrema relevancia, porque puede evitar la alteración del régimen terapéutico anticoagulante y el riesgo de un exceso de sangrado. En el presente trabajo se investiga la capacidad hemostática de la cola de fibrina como presidio hemostático en los pacientes bajo terapia anticoagulante, exponiendo los resultados de la experimentación sobre la base de un Standard de referencia del trauma quirúrgico y de la intensidad de la terapia anticoagulante.

Materiales y métodos: Han sido seleccionados 20 pacientes en terapia anticoagulante con una edad comprendida entre los 44 y 79 años, se han realizado, según las modalidades apropiadas, simples exodoncias o más complejas de dientes mono-, bi- y tri-radiculares. Tras la alveoloplastia se ha colocado una esponja de fibrina reabsorbible en forma de gel (Gingistat ${ }^{\circledR}$ ) y se ha realizado la sutura. El trauma quirúrgico ha sido clasificado por medio de una escala cuantitativa que puede expresar de forma unívoca el grado de trauma. La categoría de trauma por cada paciente se establece por la suma de los valores obtenidos de cada uno de los procedimientos unitarios. Ha sido posible evaluar el coeficiente de relación entre la intensidad del efecto anticoagulante (medido por medio del INR) y número de complicaciones hemorrágicas que se han verificado, además de entre el grado de trauma quirúrgico y el número de complicaciones que se han presentado.

Resultados y Conclusiones: Se demuestra que el utilizo terapéutico de la cola de fibrina puede ser considerado como un válido soporte por la optimización de la hemostasis en los pacientes con alteraciones de la coagulación inducidas farmacológica mente.

Palabras clave: Terapia anticoagulante, cola de fibrina, cirugía odontoestomatológica.

\section{SUMMARY}

Aim of the work: The authors emphasize how an effective emostatic method is extremely important in patients treated with anticoagulating therapy during oral surgery: it can avoid the risk of an excessive emorrhage and the necessity to alter the anticoagu- lating therapeutic regimen. The present study examines the fibrin glue emostatic ability as emostatic support in patients treated with anticoagulating therapy. It expresses the experiment results,based on standards of reference of surgical trauma and considers the anticoagulating therapy intensity.

Materials and methods: 20 patients with anticoagulation therapy have been selected within the age of 44 and 79 . Following the appropriate techniques extractions of teeth with one, two or three roots have been practiced. After the alveoloplasty a reabsorbable sponge of fibrine is placed and the suture is done. The surgical trauma has been classified by means of a quantitative scale which can express it without inaccuracy. The category of trauma for each patient is established by the total of the values obtained from each of the single procedures. It has been possible to evaluate the coefficient of relationship between the intensity of the anticoagulating effect (measured by the INR) and the number of verified hemorrhagic complications; and also between the grade of surgical trauma and the number of complications.

Results and conclusions: The authors demonstrate how the therapeutical usage of fibrin glue can be considered a valid support for the optimization of the haemostatis in patients with induced alterations of the coagulation. 
Key world: Anticoagulating therapy, fibrin glue, oral surgery.

Aceptado para publicación: marzo 2003.

* - Odontólogo Colaborador del Curso de Formación Académica para Higienistas Dentales, Facultad de Medicina y Cirurgía, Università degli Studi di Milano-Bicocca.

** Odontólogo Colaborador del Curso de Formación Academca para laurea per Igienistas Dentales, Facultad de Medicina e Cirugía, Università degli Studi di Cagliari.

*** Odontólogo y Master Periodoncia Universidad Complutense.

Taormina G, Papagna R, Carini F, Denotti G, Calzavara D. Cirugía oral en pacientes bajo terapia anticoagulante: modelo experimental para la evaluación de la eficacia de la cola de fibrina. Av. Odontoestomatol 2004; 20-3: 139146

\section{INTRODUCCIÓN}

El papel del sistema hemostático, en condiciones normales, es evitar un sangrado excesivo, y la formación de trombos. Los pasos determinantes en una trombosis arteriosa (generalmente un trombo piastrìnico) o venosa (generalmente un coágulo de fibrina) están bajo el control de una serie de sustancias y componentes endógenos plasmáticos y tisulares. Actualmente, no se ha precisado aun todo el conjunto de activadores e inhibidores ni los respectivos mecanismos de relación con la base de los fenómenos de agregación piastrínica, coagulación y fibrinolisis.

En varias situaciones clínicas está indicada una intervención terapéutica, realizada por medio de fármacos anticoagulantes, con finalidad de inducir la inhibición del sistema de la coagulación, disminuyendo la formación no deseada de trombos. En la Tabla 1 se exponen los resúmenes principales sobre los empleos clínicos de los fármacos anticoagulantes (1).

La terapia con anticoagulantes induce un frágil equilibrio entre el efecto terapéutico excesivo (riesgo hemorrágico) y el efecto terapéutico insuficiente (riesgo trombo embólico). La intensidad ideal del efecto anticoagulante, necesario para prevenir la aparición de ambos eventos (hemorragia o tromboembolia), no es todavía perfectamente conocida (2). En el 1983, la World Health Organization (WHO) sugiere la utilización de la normalización del tiempo de protrombina (PT) para el control de los anticoa-
TABLA 1. ESQUEMA TERAPÉUTICO DEL CONJUNTO DE LOS FÁRMACOS ANTICOAGULANTES (HEPARINA, CUMARINICOS)

\author{
Trombosis arterial \\ Fibrilación atrial \\ Cardiopatías \\ Embolismo cerebral \\ Prótesis vasculares \\ Valvulopatías cardíacas \\ Tromboembolismo venoso
}

gulantes orales; se trata del International Normalized Ratio (INR) (3). Parece que la intensidad adecuada a largo plazo de la terapia anticoagulante está comprendida entre 2.0 y 3.0 INR para todas las indicaciones terapéuticas, con la excepción de la prevención del riesgo embolico en pacientes con válvulas cardiacas mecánicas y con infarto de miocardio donde está indicado un INR variable entre 2.5 y 3.5.

La cirugía oral en los pacientes en terapia con anticoagulantes origina una importante cuestión: reducir de forma transitoria la intensidad de la terapia anticoagulante y exponerse al riesgo de tromboembolismo, o de seguir la terapia anticoagulante y por lo tanto exponerse al riesgo de un exceso de sangrado postoperatorio (5). Un método hemostático eficaz es de elevada importancia en estos pacientes, porque de esta manera pueden evitar suspender su terapia 
anticoagulante y el riesgo de un exceso de sangrado. La importancia de un instrumento hemostático eficaz aumenta si se toma en consideración que el sangrado postoperatorio no está relacionado con el valor de INR, cuanto más con el grado de inflamación local del tejido (6).

En los últimos años, varios investigadores se han orientado hacia un tratamiento quirúrgico que contempla la combinación de la terapia anticoagulante con el uso de instrumentos hemostáticos locales y por medio de adhesivos biológicos (7, 8, 9, 10, 11). Sin embargo pocos estudios (12) han analizado la relación entre el grado de seguridad del método hemostático, grado de trauma quirúrgico e intensidad del tratamiento anticoagulante. El resultado de estos estudios clínicos depende de la técnica quirúrgica adoptada, además de la eficacia del producto empleado como hemostático (12). El objetivo de este trabajo es evaluar la capacidad hemostática de la cola de fibrina en los pacientes que están bajo terapia anticoagulante. Los resultados de la experimentación tendrán precisos Standard de referencia al trauma quirúrgico y a la intensidad de la terapia anticoagulante, según el modelo experimental propuesto por Bodner y cols. (13). Por medio de las definiciones de estos estándar paramétricos, ha sido también posible evaluar la existencia de una relación estadística entre las distintas variables que se han examinado.

\section{MATERIAL Y MÉTODOS}

La cola de fibrina es un producto hemostático que favorece la curación de los tejidos, está compuesto por factores de la coagulación (fibrinógeno, factor XIII, trombina), por un agente antifibrinolítico (aportinina) y por cloruro de calcio. Cuando los distintos componentes de la cola de fibrina están unidos llevan a la formación de un coagulo estable y no soluble de fibrina (fig. 1). Tres días tras la aplicación sobre la lesión, se puede apreciar un tejido de granulación rico en fibroblastos, granulocitos y macrófagos; entre el $4^{\circ}$ y $7^{\circ}$ día es evidente la proliferación de las fibras de colágeno; y tras 14 días se aprecia un tejido de granulación rico en colágeno con una marcada disminución del número de los infiltrados celulares 14.

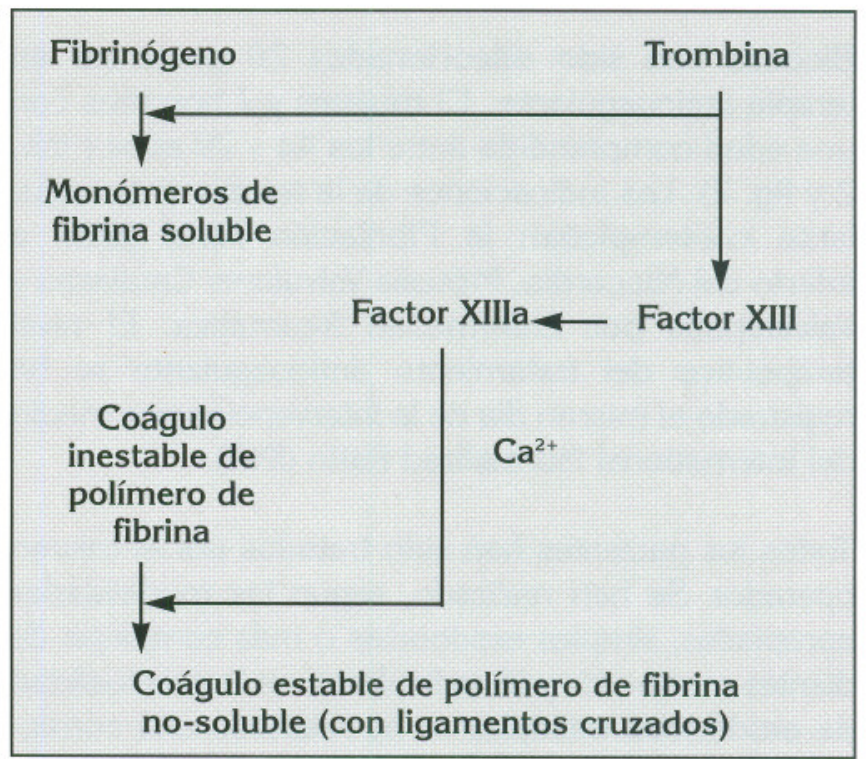

Fig. 1. Representación esquemática de la relación entre los componentes de la cola de fibrina (modificado por Dunn CJ, Goa KL. Fibrin sealant: a review of its use in surgery and endoscopy. Drugs 1999 Nov;58(5):863-86).

\section{TABLA 2. CLASES DE EDADES DE LOS PACIENTES SELECCIONADOS}

\begin{tabular}{|lcc|}
\hline Edad & $N^{\circ}$ de pacientes & Complicaciones \\
\hline $44-50$ & $2 \mathrm{f}(10 \%), 1 \mathrm{~m}(5 \%)$ & $/$ \\
$51-55$ & $2 \mathrm{f}(10 \%)$ & Ligero sangrado \\
& & $1 \mathrm{f}(5 \%)$ \\
$56-60$ & $3 \mathrm{f}(15 \%), 2 \mathrm{~m}(10 \%)$ & $/$ \\
$61-65$ & $1 \mathrm{f}(5 \%), 3 \mathrm{~m}(15 \%)$ & $/$ \\
$66-70$ & $2 \mathrm{f}(10 \%)$ & $/$ \\
$71-75$ & $2 \mathrm{f}(10 \%), 1 \mathrm{~m}(5 \%)$ & $/$ \\
$76-79$ & $1 \mathrm{f}(5 \%)$ & $/$ \\
\hline
\end{tabular}

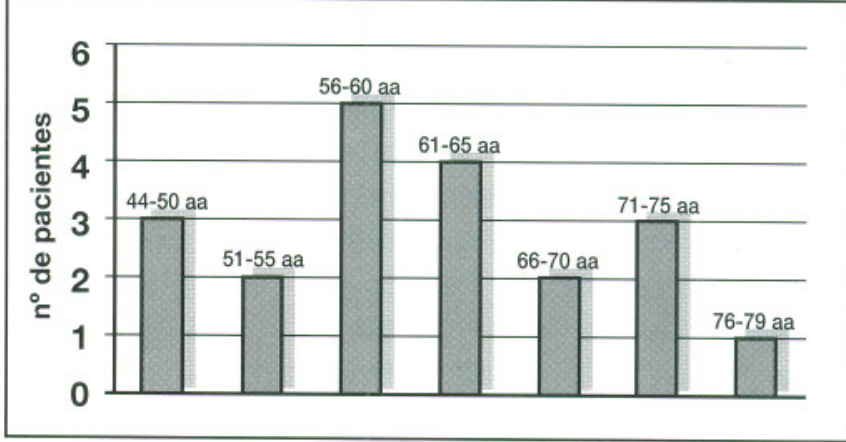

Fig. 2. Intensidad de la terapia anticoagulante y relativo porcentaje de pacientes.

En el Departamento de Cirugía Oral de la Clínica Odontoestomatológica de la Universidad de Milano 
Bicocca han sido seleccionados 20 pacientes en terapia anticoagulante, 13 mujeres y 7 hombres con una edad comprendida entre los 44 y 79 años ( tab. 2 y fig. 2). Las indicaciones de la terapia anticoagulante contemplaban la Fibrilación Atrial junta a Infarto del Miocardio, Prótesis Valvulares Cardiacas y Valvulopatía por Enfermedad Reumática. El nivel terapéutico del tratamiento anticoagulante se ha registrado el mismo día de la intervención por medio del International Normalized Ratio (INR).

Todos los pacientes han sido tratados por el mismo operador. Se han realizado, según las modalidades apropiadas, simples exodoncias o más complejas de dientes mono-, bi- y tri-radiculares; en la eventualidad de exodoncias múltiples se ha realizado una corrección plástica de los tejidos blandos residuales. Se ha establecido un protocolo de prevención de la endocarditis bacteriana según las líneas actuales del American Heart Association. Todas las intervenciones se han realizado bajo anestesia local (articaina clorhidrato al $4 \%$ con adrenalina 1:100000 o mepivacaina clorhidrato al 3\%). Tras la alveoloplastia se ha colocado una esponja de fibrina reabsorbible en forma de gel (Gingistat $\left.{ }^{\varpi}\right)$ y finalmente se ha realizado la sutura con un hilo de seda 3/0. La cola de fibrina ha sido colocada dentro de alveolo, en los márgenes de la lesión y alrededor de los puntos de sutura. Parar terminar se ha aplicado una ligera presión durante unos 3 minutos, con la finalidad de conseguir un adherencia adecuada entre los elementos de coágulo. Las suturas se han removido tras 7 días. Según el modelo experimental de Bodner y cols. (13), el trauma quirúrgico ha sido clasificado por medio de una escala cuantitativa que puede expresar de forma unívoca el grado de trauma. Esta proporciona los siguientes valores: 1 , exodoncia simple de un diente con raíz unitaria; 2 , exodoncia simple de diente biradicular; 3 , exodoncia simple de triradicular; 4 , exodoncia quirúrgica. La categoría de trauma por cada paciente se establece por la suma de los valore obtenidos de cada uno de los procedimientos unitarios.

Ha sido posible evaluar el coeficiente de relación entre la intensidad del efecto anticoagulante (medido por medio del INR) y número de complicaciones hemorrágicas que se han verificado, además de entre el grado de trauma quirúrgico y el número de complicaciones que se han presentado.
TABLA 3. VALORES DEL INR

\begin{tabular}{|lccc|}
\hline Intensidad & INR & $\begin{array}{c}N^{\circ} \text { Pacientes } \\
(\%)\end{array}$ & $\begin{array}{c}\text { Complica- } \\
\text { ciones }\end{array}$ \\
\hline Baja & $1.0-1.5$ & $1(5 \%)$ & $/$ \\
& $1.6-2.0$ & $2(10 \%)$ & $/$ \\
Media & $2.0-2.5$ & $4(20 \%)$ & $/$ \\
& $2.6-3.0$ & $5(25 \% /)$ & ligero sangrado \\
& & & $1(5 \%)$ \\
Alta & $3.0-3.5$ & $5(25 \%)$ & $/$ \\
& $3.6-4.0$ & $1(5 \%)$ & $/$ \\
& $4.0-4.5$ & $1(5 \%)$ & $/$ \\
& $4.6-5.0$ & $1(5 \%)$ & $/$ \\
\hline
\end{tabular}

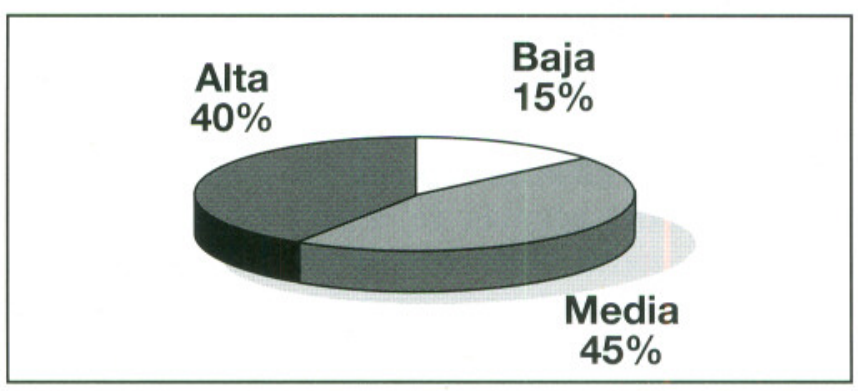

Fig. 3. Valores del INR.

\section{TABLA 4. GRADO DE TRAUMA QUIRÚRGICO}

\begin{tabular}{|lcc|}
\hline Categoría & $N^{\circ}$ de pacientes $(\%)$ & Complicaciones \\
\hline 1 & $3(15 \%)$ & $/$ \\
2 & $1(5 \%)$ & $/$ \\
3 & $2(10 \%)$ & $/$ \\
4 & $3(15 \%)$ & $/$ \\
5 & $2(10 \%)$ & $/$ \\
6 & $3(15 \%)$ & $/$ \\
7 & $2(10 \%)$ & \\
8 & $/$ & $/$ \\
9 & $1(5 \%)$ & ligero sangrado $1(5 \%)$ \\
10 & $2(10 \%)$ \\
11 & $1(5 \%)$ & $/$ \\
\hline
\end{tabular}

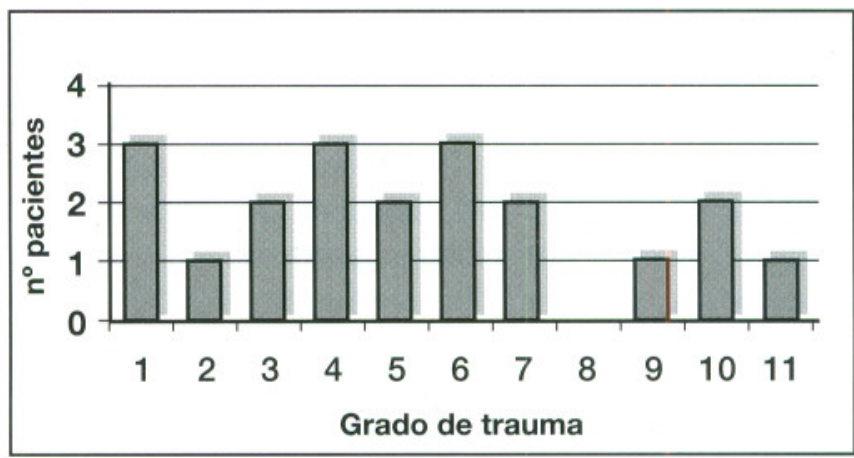

Fig. 4. Grado de trauma quirúrgico 

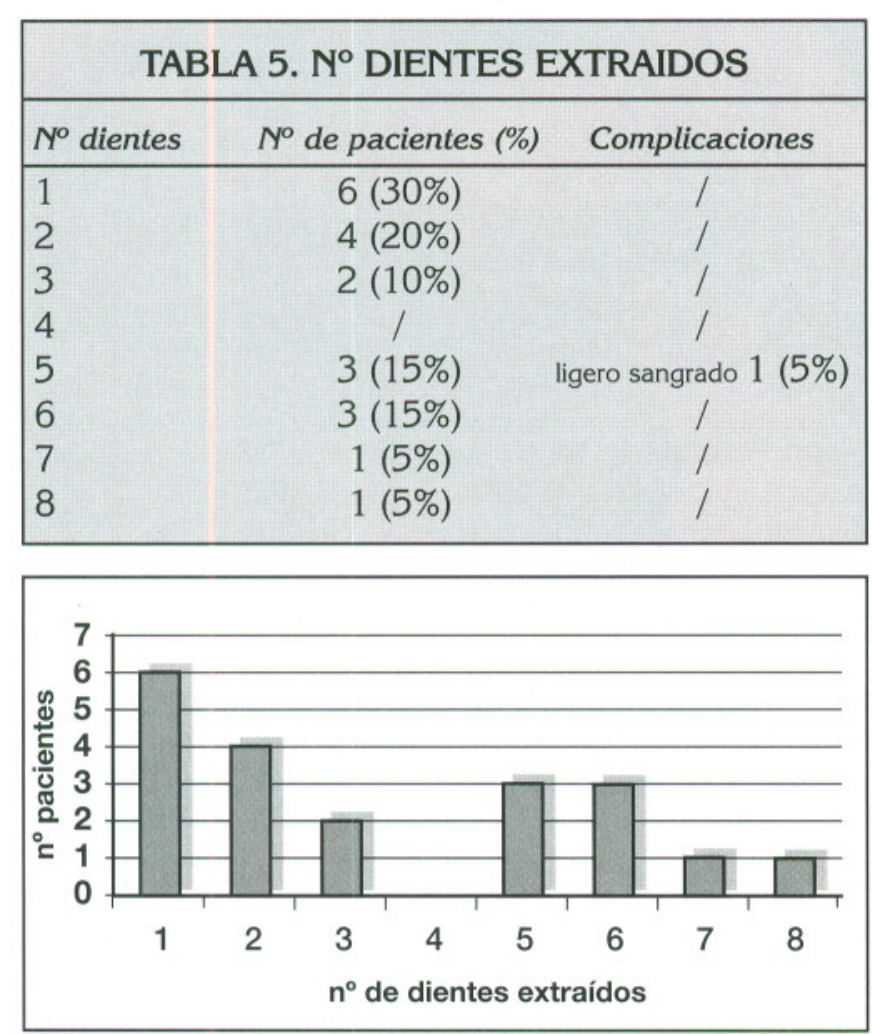

Fig. 5. Número de dientes extraídos por pacientes.

\section{RESULTADOS}

En la tabla 3 y Figura 3, donde se exponen los valores de INR registrados, se observa que 3 pacientes (15\%) presentaban un valor de INR inferior a los considerados ideales (INR $=1-2)$, con una tendencia a un insuficiente efecto terapéutico anticoagulante; 9 pacientes (45\%) presentaban un valor de INR que para muchos de ellos era compatible con un efecto anticoagulante ideal (INR $=2-3)$; 8 pacientes $(40 \%)$ presentaban un INR superior a los valores ideales (INR=3-5), mostrando una tendencia hacia un sobretratamiento anticoagulante. El valor de INR terapéutico medio ha sido de 2,8 .

Las categorías del trauma quirúrgico, expuestas en la Tabla 4 y Figura 4, comprendían 6 pacientes (30\%) de las categorías 1-3 y los remanentes 14 (60\%) estaban en las categorías 4-11. El trauma quirúrgico medio ha sido de 5,25.

El número de dientes extraidos por cada paciente aparece en la Tabla 5 y en la Figura 5, donde se puede apreciar que en 10 pacientes $(50 \%)$ se han rea- lizados las exodoncias de 3 o más dientes. La media de dientes extraídos por cada paciente ha sido 3,4.

No se han apreciado casos de severa o de larga hemorragia post-operatoria. Solo en 1 caso se ha verificado un ligero sangrado a lo largo del día siguiente la intervención; una ligera presión localizada ha sido suficiente para parar el sangrado. No se han registrado casos de curaciones complicadas o de alguna infección de la lesión quirúrgica.

Por medio del análisis estadístico del coeficiente de relación, que permite analizar la relación entre dos grupos de datos, se ha podido demostrar que, en los pacientes que están bajo terapia anticoagulante, no existe una relación estadísticamente significativa entre la entidad del efecto anticoagulante y la incidencia de complicaciones hemorrágicas ( $p=$ 0.2582). Tampoco existe una relación estadísticamente significativa entre la entidad del trauma quirúrgico y la incidencia de las hemorragias $(p=0.3667)$, ni entre el número de dientes extraídos y la incidencia de complicaciones hemorrágicas $(p=0,1603)$. No existe una relación estadísticamente significativa tampoco entre la edad del paciente y la incidencia de complicaciones hemorrágicas $(p=-$ $0,2186)$. Estos resultados se han obtenido desde las intervenciones de cirugía oral realizadas a nivel ambulatorio, por medio de $v$ instrumentos hemostáticos válidos, como la cola de fibrina asociada con esponja reabsorbible de gelatina y puntos de sutura.

\section{DISCUSIÓN Y CONCLUSIONES}

El empleo terapéutico de la cola de fibrina tiene que ser considerado como un válido instrumento por la optimización de la hemostasis en los pacientes con alteraciones de la coagulación inducidas por fármacos o congénitas, por el hecho de que el sangrado tras una cirugía oral podría ser prolongado y excesivo. (figuras 6-9) (15).

La técnica quirúrgica y la técnica de aplicación de la cola de fibrina son las dos principales variables. El fracaso en la prevención de la hemorragia causada por las maniobras de extracción han sido citadas en un estudio sobre pacientes hemofílicos (16), donde en lecho extractivo no fue realizada la sutura 


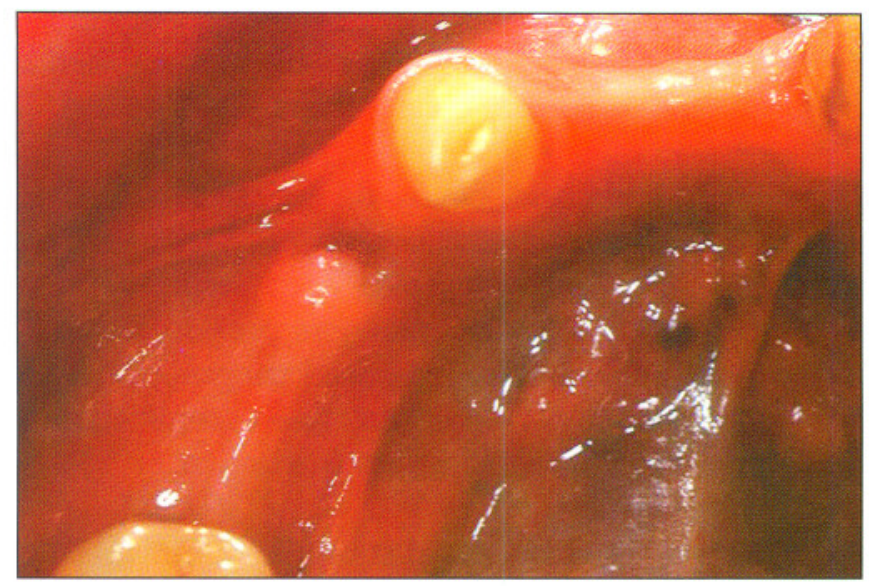

Fig. 6. En este paciente fue necesaria la exodoncia del 44 .

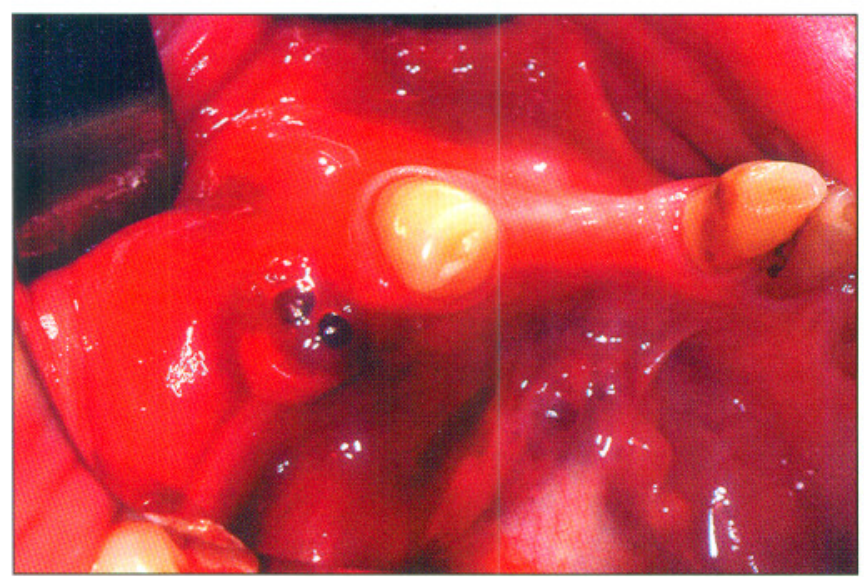

Fig. 7. Visión del alveolo inmediatamente tras la exodoncia; se puede apreciar el copioso sangrado.

y esto ha sido la causa de la dilución y de la exportación de la cola de fibrina por parte de la saliva. Además, una excesiva concentración de trombina en la formulación de la cola lleva a un retraso en la formación del coagulo, generando el riesgo potencial añadido de sangrado (17). A causa de las diferentes composiciones de las colas de fibrina que se comercializan, hay que evitar extrapolar fracasos o éxitos expresados en la literatura como válidos para todas las formulaciones de estos fármacos hemostáticos (18).

Recientes publicaciones $(19,20)$ vuelven hacerse la pregunta sobre el valor de una posible interrupción o alteración del régimen terapéutico anticoagulante en casos de cirugía oral. Está bien claro que, actuando con medidas hemostáticas locales correctas, no está justificada la alteración del régimen terapéutico anti-

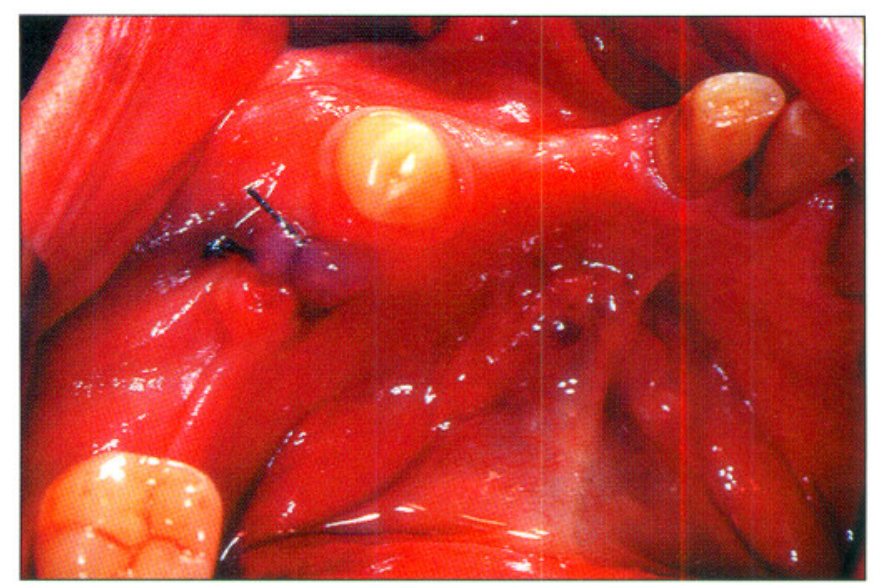

Fig. 8. Alveolo tras la aplicación de la cola de fibrina y de las suturas; se aprecia la ausencia de sangrado.

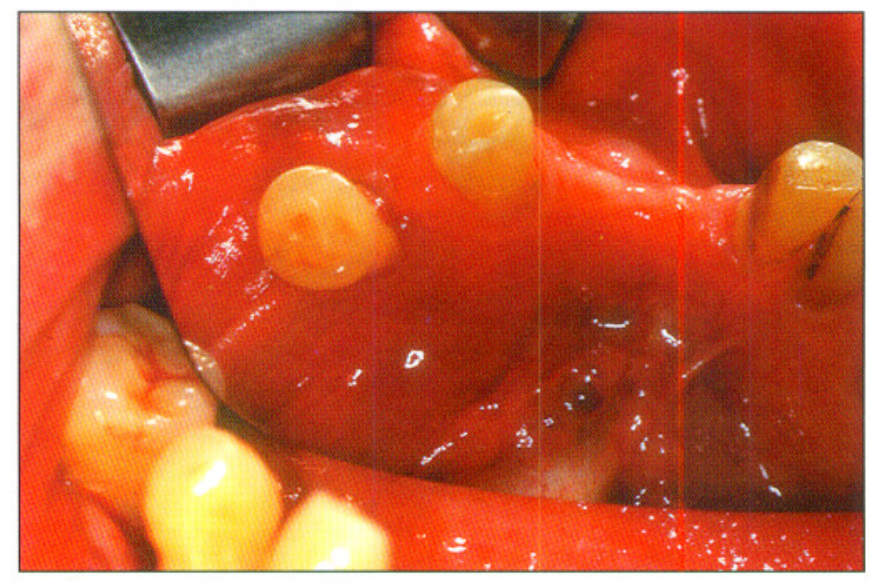

Fig. 9. La curación, 12 días tras la exodoncia.

coagulante, porque con esta línea se podría exponer el paciente al riesgo bien documentado (21), de complicaciones tromboembólicas.

En esta experimentación se ha demostrado que la entidad del trauma quirúrgico, el número de dientes extraídos (que puede ser considerado a su vez un índice indirecto del grado de trauma quirúrgico), así como la edad de los pacientes no influyen sobre el éxito de las maniobras hemostáticas con el uso de la cola de fibrina. Estos resultados tiene que ser interpretados de forma crítica teniendo en consideración que en los pacientes en terapia anticoagulante, el riesgo de complicaciones hemorrágicas aumenta de forma significativa con la edad y con el aumento de la intensidad del efecto anticoagulante, de este último el INR es válido y parece depender del tipo de anticoagulante oral (22). 
Taormina G, Papagna R, Carini F, Denotti G, Calzavara D

Cirugía oral en pacientes bajo terapia anticoagulante:

Modelo experimental para la evaluación de la eficacia de la cola de fibrina

\section{BIBLIOGRAFÍA}

1. Brody TM, Larner J, Minneman KP, Neu HC. Human Pharmacology molecular to clinical. 2nd edition, 1994, Mosby-Year Book, Inc; 269-70.

2. Azar AJ, Cannegieter SC, Deckers JW, Briet E, van Bergen PF, Jonker JJ, Rosendaal FR. Optimal intensity of oral anticoagulant therapy after myocardial infarction. J Am Coll Cardiol, 1996, May; 27 (6): 1349-55.

3. Loeliger EA, van den Besselaar AM, Lewis SM. Reliability and clinical impact of the normalization of the prothrombin times in oral anticoagulant control. Thromb Haemost 1985 Feb 18; 53 (1): $148-54$.

4. Raskob GE. Oral anticoagulant therapy. Curr Opin Hematol 1996 Sep; 3 (5): 361-4.

5. Bodner L, Weinstein JM, Baumgarten AK. Efficacy of fibrin sealant in patients on various levels of oral anticoagulant undergoing oral surgery. Oral Surg Oral Med Oral Pathol Oral Radiol Endod 1998 Oct; 86 (4): 421-4.

6. Blinder D, Martinowitz U, Ardekian L, Peleg M, Taicher S. [Oral surgical procedures during anticoagulant therapy]. Harefuah 1996 May 15; 130 (10): 681-3, 727.

7. Wepner F, Fries R, Platz H. The use of the fibrin adhesion system for local hemostasis in oral surgery. J Oral Maxillofac Surg 1982 Sep; 40 (9): 555-8.

8. Palattella G, Massi C, Corbelli V, Ruggeri B, Pignatelli N, Palattella E. [Use of lyophilized human fibrin glue "Tissucol" in oral surgery]. Dent Cadmos 1985 Apr 30; 53 (7): 65-8, 71-3.

9. Petersen JK. Clinical experience in oral surgery with human fibrin sealant. Int Dent J 1985 Dec; 35 (4): 277-9.

10. Raborn GW, Hohn FI, Grace MG, Arora BK. Tisseel, a two component fibrin tissue sealant system: report of a trial involving anticoagulated dental patients. J Can Dent Assoc 1990 Aug; 56 (8): 779-81.

11. Federici $A B$, Sacco R, Stabile F, Carpenedo $M$, Zingaro E, Mannucci PM. Optimising local therapy during oral surgery in patients with von Willebrand disease: effective results from a retrospective analysis of 63 cases. Haemophilia 2000 Mar; 6 (2): 71-7.

12. Dunn CJ, Goa KL. Fibrin sealant: a review of its use in surgery and endoscopy. Drugs 1999 Nov; 58 (5): 863-86.

13. Pfluger $\mathrm{H}$. Lysis and adsorption of fibrin sealant (Tissucol/Tisseel). In: Schlag G, Redl H, editors. Fibrin Sealant in operative medicine. General surgery and abdominal surgery. V. 6. Berlin, Heidelberg: Springer-Verlag, 1986: 39-50.

14. Beccu L, Knieriem H-J. Histologische Ergebnisse beim Menschen. In: Friedrichs O, Editor. Fibrinklebung in der Endoskopie. Berlin, Wien: Blackwell Wissenschafts, 1996: 53-66.

15. Mannucci PM. Hemostatic drugs. N Engl J Med 1998; 339 (4): 245-53.

16. Hoots K, McLeod J, Eqqers E, et al. Pilot study to evaluate efficacy of fibrin sealant (human) on hemostasis in hemophiliacs undergoing tooth extraction. Blood 1993; 82: 598.

17. Martinowitz U; Spotnitz D. Fibrin tissue adhesives. Thromb Haemost 1997; 78 (1): 661-6.

18. Martinowitz U, Schulman S. Fibrin sealant in surgery of patient with a hemorrhagic diathesis. Thromb Haemost 1995; 74 (1): 486-92.

19. Devani P, Lavery KM, Howell CJ. Dental extractions in patients on warfarin: is alteration of anticoagulant regime necessary? $\mathrm{Br} \mathrm{J}$ Oral Maxillofac Surg 1998 Apr; 36 (2): 107-11.

20. Campbell JH, Alvarado F, Murray RA. Anticoagulation and minor oral surgery: should the anticoagulation regimen be altered? J Oral Maxillofac Surg 2000 Feb; 58 (2): 131-5. 
21. Wahl MJ. Dental surgery in anticoagulated patients. Arch Intern Med 1998 Aug 10-24; 158 (15): 1610-6.

22. Van der Meer FJ, Rosendaal FR, Vandenbroucke JP, Briet E. Bleeding complications in oral anticoagulant therapy. An analysis of risk factors. Arch Intern Med 1993 Jul 12;153 (13): 1557-62

\section{CORRESPONDENCIA}

Ruggero Papagna

Cod. Fisc. PPGRGR77P01F205C

Via Taramelli 36, Bergamo

Tel. y Fax: 035249683 